\title{
Does Chemical Cross-Linking with NHS Esters Reflect the Chemical Equilibrium of Protein- Protein Noncovalent Interactions in Solution?
}

\author{
Stefanie Mädler, ${ }^{\text {a }}$ Markus Seitz, ${ }^{\text {b }}$ John Robinson, ${ }^{\mathrm{b}}$ and Renato Zenobi ${ }^{\mathrm{a}}$ \\ ${ }^{a}$ Department of Chemistry and Applied Biosciences, ETH Zurich, Zurich, Switzerland \\ ${ }^{\mathrm{b}}$ Institute of Organic Chemistry, University of Zurich, Zurich, Switzerland
}

Chemical cross-linking in combination with mass spectrometry has emerged as a powerful tool to study noncovalent protein complexes. Nevertheless, there are still many questions to answer. Does the amount of detected cross-linked complex correlate with the amount of protein complex in solution? In which concentration and affinity range is specific cross-linking possible? To answer these questions, we performed systematic cross-linking studies with two complexes, using the N-hydroxysuccinimidyl ester disuccinimidyl suberate (DSS): (1) NCoA-1 and mutants of the interacting peptide STAT6Y, covering a $\mathrm{K}_{\mathrm{D}}$ range of $30 \mathrm{nM}$ to $>25 \mu \mathrm{M}$, and (2) $\alpha$-thrombin and basic pancreatic trypsin inhibitor (BPTI), a system that shows a bufferdependent $K_{D}$ value between 100 and $320 \mu \mathrm{M}$. Samples were analyzed by matrix-assisted laser desorption/ionization mass spectrometry (MALDI-MS). For NCoA-1 $\bullet$ STAT6Y, a good correlation between the amount of cross-linked species and the calculated fraction of complex present in solution was observed. Thus, chemical cross-linking in combination with MALDI-MS can be used to rank binding affinities. For the mid-affinity range up to about $\mathrm{K}_{\mathrm{D}} \approx$ $25 \mu \mathrm{M}$, experiments with a nonbinding peptide and studies of the concentration dependence showed that only specific complexes undergo cross-linking with DSS. To study in which affinity range specific cross-linking can be applied, the weak $\alpha$-thrombin $\bullet$ BPTI complex was investigated. We found that the detected complex is a nonspecifically cross-linked species. Consequently, based on the experimental approach used in this study, chemical cross-linking is not suitable for studying low-affinity complexes with $\mathrm{K}_{\mathrm{D}} \gg 25 \mu \mathrm{M}$. (J Am Soc Mass Spectrom 2010, 21, 1775-1783) (c) 2010 American Society for Mass Spectrometry

$\mathrm{T}$ The identification and quantitation of proteinprotein interactions is the basis for understanding most cellular pathways. Numerous analytical techniques have been developed to gain insight into biochemical communication between proteins, such as circular dichroism spectroscopy (CD), isothermal titration calorimetry (ITC), nuclear magnetic resonance spectroscopy (NMR), surface plasmon resonance (SPR), light scattering, gel-filtration, or fluorescence polarization assays (FPA), to name just a few. The ideal method would yield the stoichiometry and size of a complex, as well as thermodynamic and kinetic data, and would allow differentiating between specific and nonspecific interactions over a broad affinity range. Physiologically relevant interactions have dissociation constants $\left(\mathrm{K}_{\mathrm{D}}\right)$ between $10^{-16}$ and $10^{-3} \mathrm{M}$, which is quite a demanding dynamic range for any detection method [1, 2].

Chemical cross-linking in combination with electrospray ionization (ESI) or matrix-assisted laser desorption/ ionization mass spectrometry (MALDI) mass spectrometry (MS) has emerged as a valuable tool to study

Address reprint requests to Professor R. Zenobi, Department of Chemistry and Applied Biosciences, ETH Honggerberg HCI E 329, CH-8093 Zurich, Switzerland. E-mail: zenobi@org.chem.ethz.ch protein-protein or protein-ligand interactions. Topdown or bottom-up strategies can aid to map binding interfaces using the cross-linker arm as a ruler to constrain distances [3,4]. Chemical cross-linking stabilizes noncovalent complexes, prevents their dissociation and thus allows rapid identification of interactions and determination of complex stoichiometry and mass with minimal sample consumption [5, 6]. During MALDI ionization, most of the species present in the sample appear as singly charged pseudo-molecular ions in the spectra, greatly simplifying the data interpretation for complex mixtures. In combination with novel detector technologies, the mass range has been significantly extended, to more than $1 \mathrm{MDa}$, allowing sensitive detection of, for example, immunocomplexes or protein multimers [7, 8]. Among the most common cross-linking agents are homobifunctional N-hydroxy succinimide (NHS) esters, which were introduced more than 30 years ago and shown to be highly aminereactive [9]. The chemical cross-linking step relies upon an acylation of nucleophilic residues, in particular the amine groups of lysine or the N-terminus, and hydroxyl side chains of serine, threonine, or tyrosine [10, 11], which are accessible on the exterior surface of the proteins. When working with high protein concentra- 
tions, the probability of unintentionally forming crosslinked protein multimers as a consequence of nonspecific encounters also increases. Analysis of complexes after chemical cross-linking by MALDI-MS does not yield the occupancy of a specific binding site, i.e., this analytical strategy is susceptible to artifacts due to nonspecific binding.

The specificity of chemical cross-linking with NHS esters has been demonstrated in the lower $\mu \mathrm{M}$ concentration range using immunocomplexes [7, 12]. Only specific interactions were found to undergo chemical cross-linking. For example, Nazabal et al. showed that incubation of human and bovine serum albumins with anti-human serum albumin and an NHS cross-linker yielded a significant antibody-antigen complex peak in the MALDI mass spectra only for samples containing human serum albumin [7]. Similarly, sandwich assays of bovine prion protein containing two different monoclonal antibodies were only detected for antibodies known to interact with the antigen, but not for antiglutathione-S-transferase [12]. As demonstrated for complexes containing the peptide bradykinin and the corresponding B2 receptor, the application of chemical cross-linking with homobifunctional NHS esters is limited by the availability of reactive amino acids [13]. For the given example, the lack of accessible lysine residues close to the binding domain increased the fraction of nonspecific cross-linking. Specific cross-linking was achieved by using heterobifunctional reagents containing maleimide and NHS functionality.

Previous research indicated that chemical cross-linking also has the potential to differentiate between low- and high-affinity complexes. In studies of the functional epitope of the urokinase-type plasminogen activator receptor ( $\mathrm{UPAR}$ ) interacting with the growth factor-like module of urokinase-type plasminogen activator (uPA), complexes of alanine mutants of the amino-terminal fragment of uPA (ATF) and UPAR with dissociation constants in the low to high nanomolar range were subjected to chemical cross-linking using disuccinimidyl suberate (DSS). After analysis of the radioactively labeled and cross-linked samples by SDS-PAGE and autoradiography, a semiquantitative correlation of complex levels with kinetic data obtained by surface plasmon resonance was observed, giving no indication on its absolute agreement [14, 15]. Chemical cross-linking with NHS esters in combination with MALDI-MS has been successfully applied to monitor the effect of smallmolecule ligands on the binding of a coactivator peptide (SRC1) to the human estrogen receptor $\alpha$ ligand binding domain (hER $\alpha$ LBD). A semiquantitative correlation between complex levels in solution and complex levels detected by MALDI-MS was observed [16].

So far, chemical cross-linking with NHS esters has mainly been applied to high-affinity protein-protein complexes with dissociation constants in the picomolar or low nanomolar range, such as human serum albumin and its corresponding antibody $[7,17]$ or calmodulin $\bullet$ melittin [18]. Few studies report the use of cross-linking to investigate lower affinity complexes. For example, in the case of the CRT (189-88) $\bullet$ ERp57 complex $\left(\mathrm{K}_{\mathrm{D}}=\right.$ $9.1 \pm 3.0 \mu \mathrm{M})$, chemical cross-linking was used to confirm the interaction [19]. The authors are not aware of any study featuring chemical cross-linking of lowaffinity complexes with dissociation constants in the high micromolar range or investigations dealing with the application range (in terms of $K_{D}$ ) for specific cross-linking. This work addresses the affinity dependence and the concentration range of specific chemical cross-linking with NHS esters.

A reference system of the nuclear receptor coactivator NCoA-1 PAS-B domain and the transactivation domain of the signal transducer and activator of transcription 6 (STAT6) was chosen. The complex formation is mediated by the hydrophobic face of the C-terminal domain of STAT6 with the $\alpha$-helical recognition motif LXXLL (where $\mathrm{L}$ is leucine and $\mathrm{X}$ any amino acid) [20]. In Figure 1, a ribbon diagram of NCoA-1 (V259-E367) complexed with a STAT6 derived peptide (L795-E808) is shown (PDB ID code 1OJ5). Potentially cross-linkable lysines close to the binding pocket are highlighted. Assuming a coupling of the cross-linker to the $\alpha$-amino group of L794 (not included in 1OJ5) or K804 of the STAT6 peptide, the lysines K265 and K271 of NCoA-1 should be well within reach for the hydrophobic crosslinker DSS, which has a spacer arm capable of bridging N-N distances of 5.58 to $11.42 \AA$ [21].

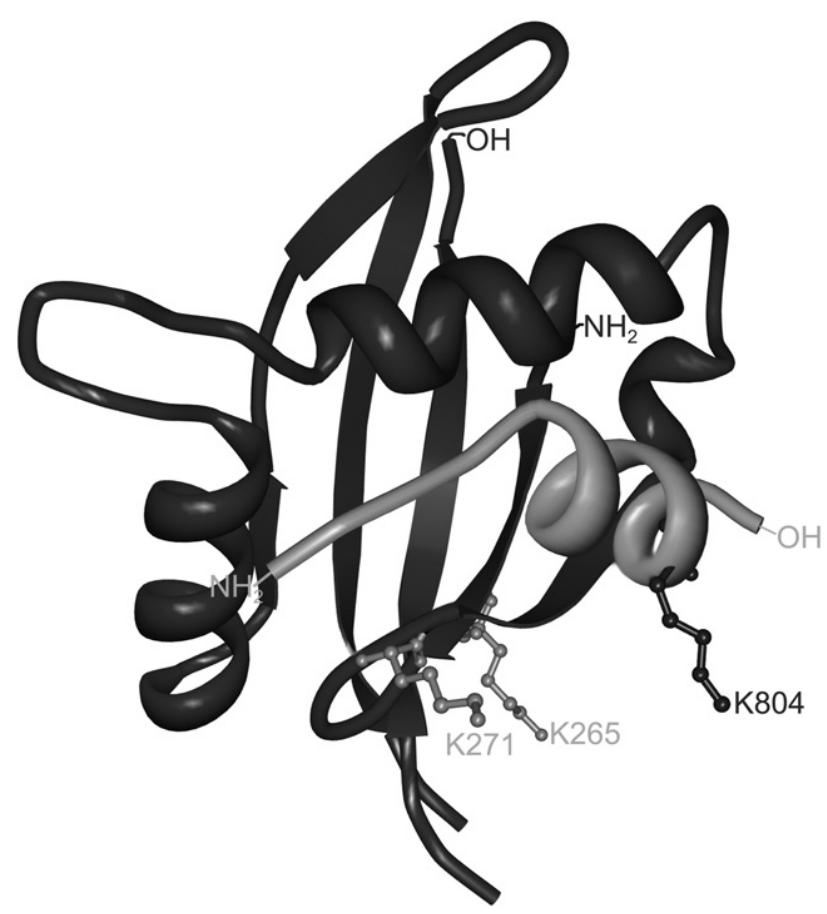

Figure 1. Ribbon diagram of the NCoA- $\bullet$ STAT6 complex (PDB ID code 1OJ5). Lysines close to the binding site that can potentially be cross-linked are highlighted for the helical STAT6-peptide (light grey ribbon, L795-E808) and the protein NCoA-1 (dark grey ribbon, V259-E367). Additionally, the N-terminus of the linear peptide Stat6Y (L794-G814) would be well within in reach of a cross-linker linked to K271. 
NCoA-1 (257-385) complexes with alanine or cyclohexyl alanine mutants of STAT6 (L794-G814) derived peptides had been investigated previously, to derive their binding affinity by a competitive FPA. The resulting $\mathrm{K}_{\mathrm{D}} \mathrm{S}$ cover three orders of magnitude $\left(\mathrm{K}_{\mathrm{D}}=0.03 \pm\right.$ $0.015 \mu \mathrm{M}$ to $\left.\mathrm{K}_{\mathrm{D}}=13.3 \pm 0.6 \mu \mathrm{M}\right)$; some mutations essentially abolished the interaction with NCoA-1 $\left(K_{D}>25 \mu M\right)$ [22]. Six different mutants were selected based on their binding affinities with NCoA-1. The main emphasis was to cover a wide range of binding affinities to investigate a possible correlation between the fraction of complex present in solution and that detected by chemical cross-linking and MALDI-MS. With the intention of extending the range of $\mathrm{K}_{\mathrm{D}} \mathrm{S}$ to above $25 \mu \mathrm{M}$ for this study, a low-affinity complex, $\alpha$-thrombin and basic pancreatic trypsin inhibitor (BPTI) with a buffer dependent $\mathrm{K}_{\mathrm{D}}(100$ to $320 \mu \mathrm{M})$ was selected and chemically cross-linked under different conditions [23].

\section{Materials and Methods}

\section{Materials}

The nuclear receptor coactivator NCoA-1 PAS-B domain $(\mathrm{Mw}=14.8 \mathrm{kDa}$, residues $257-385$ of human NCoA-1) obtained analogous to [22] was dialyzed against a phosphate buffer $\left(10 \mathrm{mM} \quad \mathrm{Na}_{2} \mathrm{HPO}_{4} /\right.$ $\mathrm{NaH}_{2} \mathrm{PO}_{4} / 150 \mathrm{mM} \mathrm{NaCl}, \mathrm{pH}$ 8.0) and the resulting $70-\mu \mathrm{M}$ solution was diluted to 10 to $30 \mu \mathrm{M}$ using the same buffer. Alanine (A) or cyclohexylalanine (Cha) mutants of a linear peptide containing STAT6 residues L794 to G814 (Mw = $2.4 \mathrm{kDa})$ were obtained by solid-phase peptide synthesis as described previously [22], dissolved in Milli-Q water (Millipore, Molsheim, France), and diluted to 10 to $30 \mu \mathrm{M}$ in the same phosphate buffer. The activity of the protein was confirmed by an FPA analogous to [22]. The STAT6 derived peptide contained a C-terminal tyrosine to aid concentration measurements by UV (STAT6Y). Oxidized bovine insulin chain $\mathrm{A}$, basic pancreatic trypsin inhibitor (BPTI) and $\alpha$-thrombin were obtained from SigmaAldrich Chemie GmbH (Buchs, Switzerland). All peptide and protein concentrations were determined by UV-absorption at $280 \mathrm{~nm}$ using a NanoDrop 1000 (Thermo Fisher Scientific, Wilmington, DE).

The cross-linker DSS was obtained from Thermo Fisher Scientific Inc. (Rockford, IL) and sinapinic acid from Sigma-Aldrich. All commercial solvents and reagents were obtained in the highest available purity and used without additional purification.

\section{Chemical Cross-Linking}

For experiments with the NCoA-1 •STAT6Y complex, $10 \mu \mathrm{L}$ of $10 \mu \mathrm{M}$ NCoA-1 protein solution were incubated with $10 \mu \mathrm{L}$ of $10 \mu \mathrm{M}$ peptide solution for $10 \mathrm{~min}$ at $2{ }^{\circ} \mathrm{C}$. To investigate the concentration dependence, 10 $\mu \mathrm{L}$ of 10,20 , or $30 \mu \mathrm{M}$ coactivator protein solutions were incubated with equimolar amounts of peptides mutated with alanine at position 794,796 , or 797 , respectively. As negative controls, $10 \mu \mathrm{L}$ of $10,20,30$, or $70 \mu \mathrm{M}$ coactivator protein solution were incubated with equimolar amounts of the nonbinding peptide oxidized bovine insulin chain $\mathrm{A}$. Ten $\mu \mathrm{L}$ of the solution were mixed with a 50-fold molar excess of DSS dissolved in dimethylformamide (DMF) at 2, 4, or $6 \mathrm{mg} / \mathrm{mL}$ in a $10 / 1$ (vol/vol) ratio. Incubation for $4 \mathrm{~h}$ in the temperature range of $2-8{ }^{\circ} \mathrm{C}$ was chosen to ensure a complete cross-linking reaction and to avoid protein denaturation. The remaining solution was treated as a control and $1 \mu \mathrm{L}$ of DMF was added. Both control and reaction solution were quenched with $5 \mu \mathrm{L}$ of $50 \mathrm{mM} \mathrm{NH}_{4} \mathrm{HCO}_{3}$ solution and afterwards purified by ZipTip $(0.6 \mu \mathrm{L} \mathrm{C} 4$ resin; Millipore, Molsheim, France). For experiments with final protein concentrations exceeding $5 \mu \mathrm{M}$, the reaction mixtures and control samples were diluted to reach a final protein concentration comparable to the concentrations of the first experiment before ZipTip. In the case of the $\alpha$-thrombin $\bullet$ BPTI complex, $\alpha$-thrombin with a final protein concentration of about $20 \mu \mathrm{M}$ was incubated with an equimolar amount or a 2- to 5-fold molar excess of BPTI in different buffers $(10 \mathrm{mM}$ $\mathrm{Na}_{2} \mathrm{HPO}_{4} / \mathrm{NaH}_{2} \mathrm{PO}_{4}$ at $\mathrm{pH} 8.0$ with no additional salt, $145 \mathrm{mM}$ tetramethylammonium chloride/5 mM NaCl or $150 \mathrm{mM} \mathrm{NaCl}$ ) for $15 \mathrm{~min}$. The high protein concentration was carefully optimized to minimize nonspecific protein-peptide gas-phase adducts and to obtain reasonable protein peak signals in the mass spectra. However, the absolute concentration of $\alpha$-thrombin has little influence on the relative amount of protein-peptide complex. Nine $\mu \mathrm{L}$ of the solution were mixed with a 30 -fold molar excess of DSS in a 10/1 (vol/vol) ratio at room-temperature for different reaction times. For this experiment, a lower excess had to be chosen for practical reasons: at the selected peptide concentrations of up to $100 \mu \mathrm{M}$, the cross-linker concentration had to be quite high to maintain a 1:10 volume ratio of proteinpeptide:XL. However, at such high concentrations, solubility issues occurred when adding the cross-linker to the aqueous protein solution. No purification was applied.

\section{Mass Spectrometric Detection}

Two commercial MALDI-TOF mass spectrometers equipped with a previously described [24] high-mass detector (HM1; CovalX AG, Zurich, Switzerland) were employed: a Reflex III (Bruker Daltonics GmbH, Bremen, Germany), which was used for the measurements with NCoA-1 and STAT6Y, and an Axima CFR (Shimadzu/ Kratos, Manchester, UK) for the measurements for BPTI and $\alpha$-thrombin. Measurements were performed in the linear positive ion mode using standard instrument settings. Ionization was initiated by using a nitrogen laser $(337 \mathrm{~nm}$ ) with pulse energies slightly above the threshold for ion production. Each mass spectrum was the average of 100 to 200 laser shots obtained at random 
sample positions. Sinapinic acid was used as a matrix, dissolved at $10 \mathrm{mg} / \mathrm{mL}$ in water/acetonitrile/TFA $(49.95 / 49.95 / 0.1, \mathrm{vol} / \mathrm{vol} / \mathrm{vol})$. In the case of NCoA1•STAT6Y, the matrix solution was mixed with the quenched and purified reaction mixture in a $1 / 1$ (vol/ vol) ratio. For $\alpha$-thrombin $\bullet$ BPTI, the samples were directly mixed with the matrix in a $1 / 1$ (vol/vol) ratio. One $\mu \mathrm{L}$ of the mixture was spotted on a stainless steel plate and allowed to dry under ambient conditions. All mass spectra were baseline-corrected and smoothed using a Savitzky-Golay algorithm. Peak integrals were calculated by in-house developed software or OriginPro 8 (Hearne Scientific Software, Chicago, IL, USA).

\section{Results and Discussion}

\section{Effect of the Binding Affinity on the Efficiency of Chemical Cross-linking}

To first evaluate whether the binding affinity has any effect on the fraction of cross-linked complex detected, the coactivator protein NCoA-1 was incubated with individual alanine or cyclohexylalanine mutants of STAT6-derived peptides, with binding affinities covering three orders of magnitude. The peptides selected and the corresponding dissociation constants for the complex with the coactivator protein determined at 298 $\mathrm{K}$ by Seitz et al. using a competitive FPA [22] are listed in Table 1. Given that all mutants form 1:1 complexes with NCoA-1 at the same binding site and have the same amount of cross-linkable primary amino groups, this system is very well suited to study the effect of binding affinity on chemical cross-linking.

Since the experiments were conducted at temperatures of about $\mathrm{T}=278 \pm 3 \mathrm{~K}$ to avoid protein degradation, the $K_{D}$ values had to be adapted to the utilized reaction conditions using the integrated van't Hoff eq 1 :

$$
\mathrm{K}_{\mathrm{D}}^{\mathrm{T}}=\mathrm{K}_{\mathrm{D}}^{298 \mathrm{~K}} \cdot \mathrm{e}^{\frac{\Delta_{\mathrm{R}} \mathrm{H}_{298 \mathrm{~K}}^{0}}{\mathrm{R}}}\left(\frac{1}{\mathrm{~T}}-\frac{1}{298 \mathrm{~K}}\right)
$$

with $\mathrm{R}$ being the universal gas constant. For the standard reaction enthalpy $\Delta_{\mathrm{R}} \mathrm{H}_{298 \mathrm{~K}}^{0}$, a value of $-7.63 \pm 0.13$ $\mathrm{kcal} \mathrm{mol}^{-1}$ determined by ITC measurements [22] was assumed. According to eq 1, the equilibrium is shifted towards the product side with decreasing temperature in the case of an exothermic reaction $(\Delta \mathrm{H}<0)$. Therefore, higher binding affinities, i.e., lower $\mathrm{K}_{\mathrm{D}} \mathrm{s}$ and higher fractions of complex, are expected for a reaction temperature of $278 \mathrm{~K}$ compared with $298 \mathrm{~K}$. The calculated values of $\mathrm{K}_{\mathrm{D}}^{278 \mathrm{~K}}$ are listed in Table 1 as well.

The protein-peptide complexes were chemically cross-linked with the homobifunctional cross-linker DSS for $4 \mathrm{~h}$. After quenching and purification, the cross-linked samples were analyzed by MALDI-ToFMS. A CovalX high mass detector was used for all experiments to avoid detector saturation with low mass compounds and to ensure similar detection efficiency over the entire $m / z$ range. In comparison with mass spectra of control samples without any cross-linker, a significant amount of cross-linked protein-peptide complex with a mass of $18 \mathrm{kDa}$ was detected for all derivatives. In the control spectra, a peak at $m / z 14,800$ corresponding to ionized NCoA-1 was observed. However, additional nonspecific gas-phase clusters of NCoA-1•STAT6Y occurred in some spectra with a maximum intensity of about $15 \%$ (data not shown). The mass spectra of the cross-linked sample contained a slightly shifted NCoA-1 peak (due to attached crosslinker molecules whose other ends were hydrolyzed or linked to another residue within the same molecule) and a cross-linked NCoA-1 $\bullet$ STAT6Y complex. Additionally, signals corresponding to $1: 2$ and 1:3 complexes were observed in some spectra. Since nonspecific gasphase clusters did also occur in the mass spectra for the solutions without cross-linker, these peaks are assumed to be nonspecific gas-phase clusters as well. Singly charged dimer ions of NCoA-1 were observed in low intensity. Since dimerization of NCoA-1 has not been reported, and no increase in intensity in the mass spectra for the cross-linked samples was detected, the dimer is probably formed by nonspecific gas-phase clustering. Figure 2a contains MALDI mass spectra of all cross-linked NCoA-1 $\bullet$ STAT6Y complexes. In general, a fairly good correlation between the binding affinity and the amount of cross-linked complex was found. For high-affinity complexes, a high amount of cross-linked species was detected, whereas the lowaffinity complexes gave only a low amount of crosslinked species. Even for the complex with peptide P797A (proline substituted by alanine, $\mathrm{K}_{\mathrm{D}}^{278 \mathrm{~K}}>10$

Table 1. Selected STAT6Y (L794-G814) mutants and corresponding binding affinities with NCoA-1

\begin{tabular}{llccc}
\hline Name & \multicolumn{1}{c}{ Sequence } & Mw exact & $\mathrm{K}_{\mathrm{D}}$ in $\mu \mathrm{M}(298 \mathrm{~K})^{\mathrm{a}}$ & $\mathrm{K}_{\mathrm{D}}$ in $\mu \mathrm{M}(278 \mathrm{~K})^{\mathrm{b}}$ \\
\hline \hline L806Cha & LLPPTEODLTKLChaLEGQGESGY & 2440.27 & $0.03 \pm 0.015$ & $0.01 \pm 0.38$ \\
O800A & LLPPTEADLTKLLLEGQGESGY & 2343.22 & $0.11 \pm 0.02$ & $0.04 \pm 0.38$ \\
P796A & LLAPTEODLTKLLLEGQGESGY & 2374.23 & $0.39 \pm 0.04$ & $0.15 \pm 0.39$ \\
L805A & LLPPTEODLTKALLEGQGESGY & 2358.20 & $3.44 \pm 0.2$ & $1.36 \pm 0.65$ \\
L794A & ALPPTEODLTKLLLEGQGESGY & 2358.20 & $13.3 \pm 0.6$ & $5.3 \pm 1.7$ \\
P797A & LLPATEQDLTKLLLEGGESGY & 2374.23 & $>25$ & $>10 \pm 1$ \\
\hline
\end{tabular}

Mutated amino acids are marked in bold. The peptide names correspond to the mutated amino acid. The normal amino acid code is used; Cha = L-cyclohexylalanine.

${ }^{a} K_{D}$ values determined by a competitive FPA at $298 \mathrm{~K}$ were taken from reference [22].

${ }^{\mathrm{b}} \mathrm{K}_{\mathrm{D}}$ values at $278 \mathrm{~K}$ were calculated using the van't Hoff equation and thermodynamic data from [22]. 

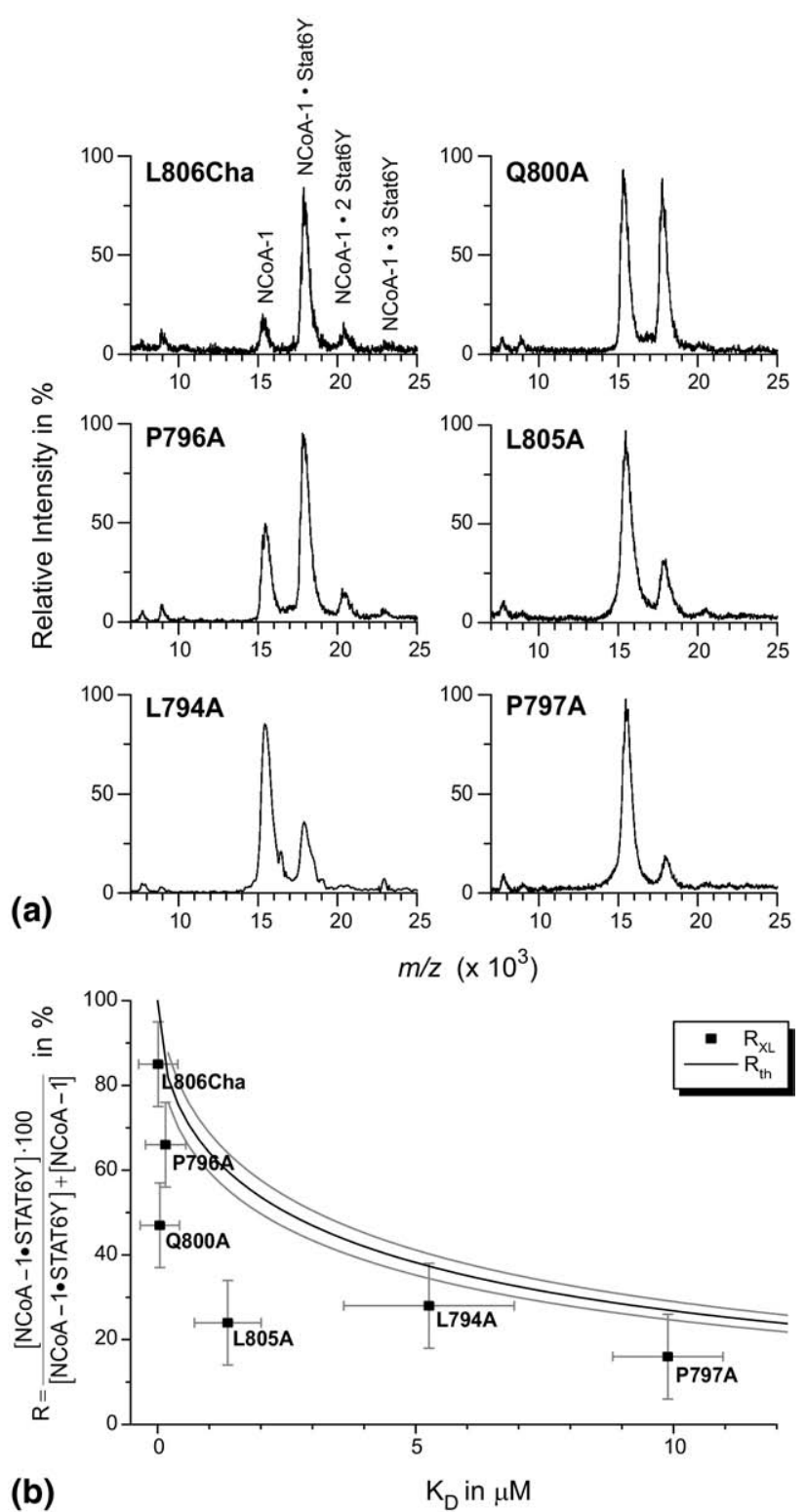

Figure 2. (a) Effects of binding affinity on the relative amount of cross-linked protein-peptide complex as determined with MALDI-MS after cross-linking; (b) Comparison with theoretically calculated values for the solution phase equilibrium based on $\mathrm{K}_{\mathrm{D}}^{278 \mathrm{~K}}$ values specified in Table 1 . For the calculations, a dissociation constant $\mathrm{K}_{\mathrm{D}}^{278 \mathrm{~K}}=10 \mu \mathrm{M}$ was assumed for the peptide P797A. For $R_{\mathrm{XL}}, \mathrm{y}$ error bars correspond to an estimated error of $\Delta \mathrm{R}=10 \%$, and $\mathrm{x}$ error bars were calculated assuming $\mathrm{c}_{\mathrm{NCOA}-1}=\mathrm{c}_{\mathrm{Stat} 6 \mathrm{Y}}=5 \pm$ $0.5 \mu \mathrm{M}$. The error of $\mathrm{R}_{\mathrm{th}}$ is shown as light grey curve.

$\mu \mathrm{M})$, the chemical cross-linking procedure yielded a significant amount of a protein-peptide adduct, which suggests the formation of a specifically stabilized complex.

The areas of the cross-linked complexes were normalized by the total peak area of the coactivator protein, $\mathrm{R}_{\mathrm{XL}}=100 \times[\mathrm{NCoA}-1 \bullet \mathrm{STAT} 6 \mathrm{Y}] /([\mathrm{NCoA}-1 \bullet \mathrm{STAT} 6 \mathrm{Y}]+$ [NCoA-1]), neglecting the 1:2 and 1:3 complexes. The fraction of cross-linked complex, $\mathrm{R}_{\mathrm{XL}}$, calculated in this fashion was compared with the theoretically calculated fraction of protein-peptide complex in solution, $\mathrm{R}_{\mathrm{th}}$, based on the known $K_{\mathrm{D}}$ values and the protein and peptide concentrations determined by UV measurements. $R_{\text {th }}$ was estimated using eqs 2 and 3 below. The dissociation constant $K_{D}$ is defined as

$$
\mathrm{K}_{\mathrm{D}}=\frac{[\mathrm{A}]_{\mathrm{eq}} \cdot[\mathrm{B}]_{\mathrm{eq}}}{[\mathrm{A} \bullet \mathrm{B}]_{\mathrm{eq}}}
$$

where $[\mathrm{A}]_{\mathrm{eq}},[\mathrm{B}]_{\mathrm{eq}}$, and $[\mathrm{A} \bullet \mathrm{B}]_{\mathrm{eq}}$ are the $\mathrm{NCoA}-1$, the STAT6Y and the NCoA-1 $\bullet$ STAT6Y complex concentrations at equilibrium, respectively. With $[\mathrm{A}]_{\mathrm{eq}}=[\mathrm{A}]_{0}-$ $[\mathrm{A} \bullet \mathrm{B}]_{\mathrm{eq}}$ and $[\mathrm{B}]_{\mathrm{eq}}=[\mathrm{B}]_{0}-[\mathrm{A} \bullet \mathrm{B}]_{\mathrm{eq}}$, where $[\mathrm{A}]_{0}$ and $[\mathrm{B}]_{0}$ are the total concentrations of NCoA-1 and STAT6Y used, the fraction of protein-peptide complex $R_{t h}$ at equilibrium can be calculated with eq 3 :

$$
\begin{aligned}
\mathrm{R}_{\mathrm{th}}= & \frac{100}{[\mathrm{~A}]_{0}}\left(\frac{[\mathrm{A}]_{0}+[\mathrm{B}]_{0}+\mathrm{K}_{\mathrm{D}}^{278 \mathrm{~K}}}{2}\right. \\
& \left.-\left(\frac{\left([\mathrm{A}]_{0}+[\mathrm{B}]_{0}+\mathrm{K}_{\mathrm{D}}^{278 \mathrm{~K}}\right)^{2}}{4}-[\mathrm{A}]_{0} \cdot[\mathrm{B}]_{0}\right)^{\frac{1}{2}}\right)
\end{aligned}
$$

A comparison of $R_{t h}$ and $R_{X L}$ is shown in Figure $2 b$. For most of the peptides, less complex was detected compared with the theoretical value. There can be different reasons for this behavior: (1) the protein may be partially inactive due to addition of the cross-linker dissolved in DMF and (2) the efficiency of chemical crosslinking may not be sufficient to stabilize all complexes present in solution. For instance, a reaction of a partially hydrolyzed cross-linker with the protein or peptide at a position close to the binding interface will not yield a cross-linked complex and thus decreases the observed fraction of cross-linked complex. Nevertheless, a reasonable agreement of the trend of $R_{X L}$ as a function of $K_{\mathrm{D}}$ with that of $\mathrm{R}_{\mathrm{th}}$ was found.

We also estimated error bars based on the following uncertainties: for the concentration measurement by UV, the error resulting from the sum of absorbance accuracy and reproducibility of the NanoDrop is about $10 \%$. Additionally, an estimated absorption coefficient $\varepsilon$ (http://expasy.org/) was used. Although only a minimum laser power at the detection limit and a final protein concentration of $5 \mu \mathrm{M}$ was used to obtain the spectra of the control and the cross-linked samples, nonspecific adduct ions could not be avoided. These aggregates of simultaneously desorbed molecular species are formed as a consequence of clustering in the expanding desorption plume and do not reflect the binding affinities in solution [25]. For the control samples without cross-linker, protein-peptide adducts were observed in some cases, with a relative peak intensity up to $15 \%$ (data not shown). For the mass spectra of cross-linked samples, cross-linked complexes aggregated with one or two additional STAT6Y peptides occurred from time to time (Figure 2a). Because of this adduct formation, quantitative analysis of the fraction of cross-linked complex by MALDI-MS is slightly ham- 
pered. Based on measured standard deviations for the relative complex amount and the presence of nonspecific gas-phase adducts, an estimated error of $10 \%$ was used for $R_{X L}$ for all peptides, since the actual error is probably larger than merely the standard deviations.

For MALDI mass spectrometry, the signal intensity of any species is highly dependent on its ionization efficiency, which may differ for a protein and its corresponding protein-ligand complex. If the protein-ligand complexes have comparable ionization efficiencies and are still detectable, a semiquantitative ranking of binding affinities should still be possible. In the case of NCoA-1 $\bullet$ STAT6Y, normalized peak intensities of the protonated peptide were compared in the MALDI mass spectra for each peptide using L806Cha as an internal standard. Within error, no substantial difference in peak intensity was observed (data not shown). Thus, comparable ionization efficiencies of the NCoA-1 $\bullet$ STAT6Y complexes are expected.

The $R_{X L}$ values of L794A and P797A exhibited a closer match between measured and theoretical complex amounts for the mutants. Two main reasons are believed to be responsible for that: (1) since nonspecific gas-phase clusters occurred in the control spectra (without cross-linker) with a relative intensity of up to $15 \%$, an overlap of cross-linked complex peaks and nonspecific gas-phase adducts is likely. The influence of unspecific adducts plays a more important role for the low-affinity complexes since the relative error of $R_{X L}$ based on nonspecific clusters is much higher than for the high-affinity complexes; (2) the peaks of the protein and the cross-linked protein-peptide complex are not perfectly resolved. Since the integration limits were chosen at the minimum intensity between these peaks, the amount of cross-linked complex is probably overestimated for the low-affinity complexes.

Nevertheless, the observed correlations between the binding affinity and the amount of cross-linked complex in the present study and previous investigations led to the assumption that chemical cross-linking in combination with MALDI-MS is a valuable tool to rank binding affinities with dissociation constants in the nanomolar and low micromolar range.

\section{Considerations on the Disturbance of the Chemical Equilibrium}

Preferably, a method for the analysis of protein-protein interactions would yield not only relative but also absolute complex levels to provide thermodynamic parameters such as dissociation constants. Our data revealed lower complex levels than predicted by solution phase equilibria. In another study dealing with the domain interplay of UPAR, the detected amount of cross-linked UPAR-ATF complex exceeded the level predicted from the equilibrium binding situation after 15 min cross-linking reaction at pH 7.4 [26]. In contrast, Bich et al. detected complex levels of about $90 \%$ of the predicted values on average after chemical cross-linking with the amine reactive homobifunctional cross-linker $1,1^{\prime}$-(suberoyldioxy)bisazabenzotriazole (SBAT) and MALDI-MS detection [27]. The question arises whether the chemical stabilization of the noncovalent complex can disturb the solution phase equilibrium and, if so, under which conditions a shift could be expected.

The NHS ester does not only react with $\mathrm{A} \bullet \mathrm{B}$, but also with the unbound $\mathrm{A}$ and $\mathrm{B}$ and thus changes the concentrations of free $[\mathrm{A}]_{\mathrm{eq}}$ and $[\mathrm{B}]_{\mathrm{eq}}$ as well (see Scheme 1). To discuss potential equilibrium shifts, the following simplifications were made: (1) the crosslinking rates for $\mathrm{A}, \mathrm{B}$, and $\mathrm{A} \bullet \mathrm{B}$ are assumed to be similar and to follow pseudo-first-order kinetics $\left(\mathrm{k}_{\mathrm{X} \mathrm{L}}^{1}=\mathrm{k}_{\mathrm{X} \mathrm{L}}^{2}=\mathrm{k}_{\mathrm{XL}}^{3}\right)$, (2) only reaction sites close to the binding pocket with a similar number of reactive amino acids for all binding partners are considered, and (3) hydrolysis of the second end of a cross-linker bound to free A or B is assumed to be complete before an appropriate binding partner is encountered $\left(\mathrm{k}_{\mathrm{on}}^{2}=0\right.$ in Scheme 1). Assumption (3) was confirmed with experimental data. Ten min of separated cross-linking of NCoA-1 and STAT6Y before mixing were sufficient to decrease the amount of cross-linked complex to a minimum after combining the two solutions (data not shown). Based on these assumptions, the numerator of eq 2, i.e., the product of $[\mathrm{A}]_{\mathrm{eq}}$ and $[\mathrm{B}]_{\mathrm{eq}}$, would decrease faster than the denominator. According to Le Chatelier's principle, the system would try to adjust the concentrations back to the equilibrium conditions by enhanced formation of reactants, i.e., dissociation of the complex.

$\mathrm{K}_{\mathrm{D}}$ equals $\mathrm{k}_{\text {off }} / \mathrm{k}_{\text {on }}$, where $\mathrm{k}_{\text {off }}$ and $\mathrm{k}_{\text {on }}$ are the kinetic constants for dissociation and association, respectively. Consequently, high-affinity complexes should be less affected by the enhanced dissociation reaction due to their low $\mathrm{k}_{\text {off }}$, since the re-establishment of the equilibrium conditions would last longer. In the case of low-affinity complexes, the high $\mathrm{k}_{\text {off }}$ values would

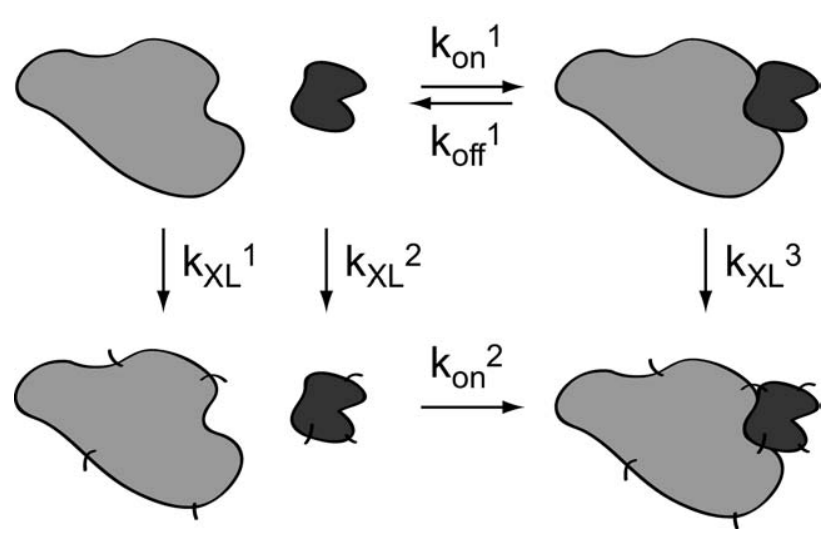

Scheme 1. Simplified scheme of reactions occurring during cross-linking of a protein-peptide complex; $\mathrm{k}_{\mathrm{XL}}$ designates the global kinetic constants of the reactions with the cross-linking agent, $\mathrm{k}_{\text {on }}$ indicates an association reaction, and $\mathrm{k}_{\text {off }}$ the dissociation of the noncovalent complex. 
allow a fast dissociation reaction and thus a lower fraction of cross-linked complex would be detected compared to the original equilibrium conditions. However, when using very efficient cross-linkers with crosslinking rate constants larger than $\mathrm{k}_{\text {off }}$ and high crosslinker excess, this concentration shift would probably not be significant, as we suppose for our data. To generate cross-linked complex levels exceeding the theoretical complex amounts, low cross-linker hydrolysis rates leading to $\mathrm{k}_{\mathrm{on}}^{2}>0$ or $\mathrm{k}_{\mathrm{XL}}^{3} \gg \mathrm{k}_{\mathrm{XL}}^{1}, \mathrm{k}_{\mathrm{XL}}^{2}$ would be required. In summary, we only would expect major deviations of the detected cross-linked complex levels from the solution phase state for complexes without reactive amino acids close to the binding pocket, for high complex dissociation rates, or for special reaction conditions.

Consequently, the best agreement with solution phase levels should occur for extremely efficient crosslinkers with high reaction speed, high reactivity towards different amino acids, and sufficient compatibility with MALDI-MS. These requirements would be partially fulfilled with the recently introduced homobifunctional cross-linker SBAT [28]. The presence of esterified 1-hydroxy-7-azabenzotriazoles as reactive groups generated about $30 \%$ more cross-linked complex, and resulted in reaction rates 10 times higher compared with DSS. In experiments comparing reaction yields of small peptides bearing only one reactive group, SBAT showed higher reactivity towards tyrosine than DSS. However, a comparable specificity to that of DSS was observed for the cross-linking of glutathione-S-transferase (GST) dimer at $5 \mu \mathrm{M}$ concentration in the presence of 3 -fold molar excess of bovine ubiquitin.

\section{Specificity as a Function of Concentration}

Since the cross-linker reacts simply with reactive amino acid side chains in close proximity and does not differentiate whether the reaction partners are participants in a specific noncovalent interaction, nonspecific crosslinking of proteins is possible. As the concentrations of the biomolecules increase, the probability of a crosslinking reaction between two nonbinding molecules increases.

To prove that the observed cross-linked NCoA$1 \bullet$ STAT6Y species is a specific complex, and to investigate the concentration range of this specificity, three different peptide mutants were chosen and their reaction behavior with the chemical cross-linker DSS was investigated for three different protein concentrations, maintaining a 1:1 protein:peptide ratio. The relative amount of cross-linked complex $\mathrm{R}_{\mathrm{XL}}$ was determined as described above and compared with the $R_{\text {th }}$ values of the relative protein complex amount in solution. The resulting concentration dependence is depicted in Figure 3. Again, a fairly good correlation between measured and theoretical values was observed. As expected, P797A, having the lowest affinity, showed a slightly lower ratio of experimental versus theoretical
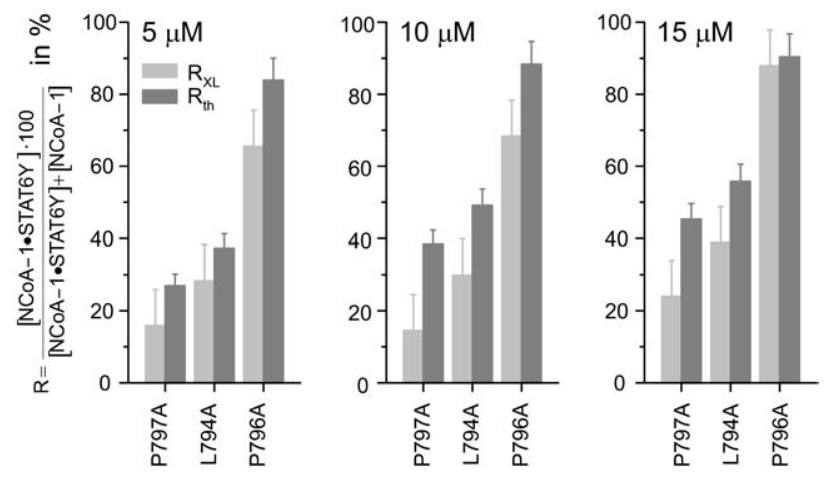

Figure 3. Effects of different NCoA-1 $\bullet$ STAT6Y concentrations on the amount of cross-linked complex detected by MALDI-MS for the peptides P797A $\left(\mathrm{K}_{\mathrm{D}}^{278 \mathrm{~K}}>10 \pm 1 \mu \mathrm{M}\right), \mathrm{P} 796 \mathrm{~A}\left(\mathrm{~K}_{\mathrm{D}}^{278 \mathrm{~K}}=0.15 \pm\right.$ $0.39 \mu \mathrm{M})$, L794A $\left(\mathrm{K}_{\mathrm{D}}^{278 \mathrm{~K}}=5.3 \pm 1.7 \mu \mathrm{M}\right)$ : Comparison of relative amounts of cross-linked complex $\mathrm{R}_{\mathrm{XL}}$ derived from the MALDI mass spectra of the reaction mixtures (light grey columns) with theoretically calculated values for the solution phase equilibrium based on $K_{D}^{278 K}$ (dark grey columns) $R_{t h}$. The concentrations given on the top correspond to the resulting protein concentration in the equimolar protein-peptide mixture. For $\mathrm{R}_{\mathrm{XL}}$, an absolute error of $\Delta \mathrm{R}=10 \%$ was estimated. Error bars for $\mathrm{R}_{\mathrm{th}}$ were calculated with the Gaussian law of errors.

complex amounts, since the actual $\mathrm{K}_{\mathrm{D}}^{278 K}$ exceeds the value of $10 \mu \mathrm{M}$ assumed for the calculations. We suppose that with increasing concentration the amount of cross-linked species that represent nonspecific interactions increases. However, the slope of the measured values is (within error bars) not significantly larger than the slope of the theoretical values. Thus, the amount of nonspecifically cross-linked species can be neglected and the specificity of chemical cross-linking can be extended to a concentration of $15 \mu \mathrm{M}$ for this system.

\section{Negative Control Experiments with a Nonbinding Peptide}

As a negative control, the reaction behavior of the cross-linker and NCoA-1 in the presence of a nonbinding peptide was tested as well. Oxidized insulin chain A was selected as a reference compound, since its molecular weight of $2.5 \mathrm{kDa}$ and thus its diffusion coefficient is quite similar to that of the mutated peptides of STAT6Y. Mixtures of protein and peptide in a 1:1 M ratio were subjected to chemical cross-linking and analyzed by MALDI-MS. As illustrated in Figure 4, no significant amount of a cross-linked complex between the coactivator protein and the insulin peptide was observed. Visible adduct peaks have about the same intensity as in the control spectra (shown in grey). Even at higher protein and peptide concentrations $(35 \mu \mathrm{M})$, no significant amount of cross-linked complex was detected. Consequently, chemical cross-linking requires a specific interaction between the coactivator protein NCoA-1 and a peptide. No unspecific cross-linking occurred under the described reaction conditions. 


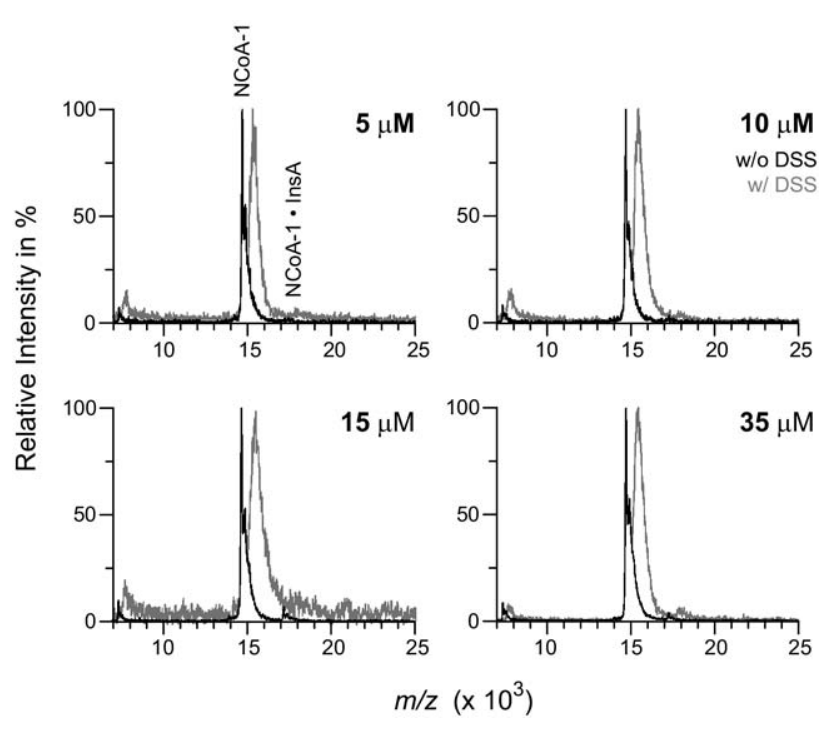

Figure 4. Effects of NCoA-1 concentrations on the amount of cross-linked complex detected by MALDI-MS for the nonbinding peptide oxidized insulin chain A. The grey curves correspond to the MALDI mass spectra of control mixtures without any crosslinker. The grey curves are the MALDI mass spectra of the reaction mixture containing a 50-fold molar excess of cross-linker. No cross-link between NCoA-1 and the insulin peptide was observed after an incubation time of $4 \mathrm{~h}$.

\section{Cross-Linking of Weak Complexes}

As discussed above, a noncovalent complex of NCoA$1 \bullet$ STAT6Y with $\mathrm{K}_{\mathrm{D}}^{298 \mathrm{~K}}>25 \mu \mathrm{M}$ could be chemically stabilized and detected by MALDI-MS with no indication of nonspecific cross-linking. However, a number of cellular processes involve even weaker complexes. Physiologically relevant interactions as weak as $\mathrm{K}_{\mathrm{D}} \approx$ $10^{-4}$ to $10^{-3} \mathrm{M}$ are largely overlooked by most analytical techniques such as ITC, X-Ray crystallography, or SPR. One approach to detect such fragile complexes is NMR, as reviewed in reference [2]. To test whether chemical cross-linking with MALDI-MS could be used to specifically detect weak protein-protein interactions, experiments with the weak $\alpha$-thrombin $\bullet$ BPTI complex were conducted. BPTI $(m / z 6500)$ binding to $\alpha$-thrombin $(m / z 36,000)$ is dependent on the sodium ion concentrations, which affect the conformational state of $\alpha$-thrombin [29]. Whereas a concentration of $150 \mathrm{mM} \mathrm{NaCl}$ yields a $\mathrm{K}_{\mathrm{D}}$ of $100 \mu \mathrm{M}$, the dissociation constant decreases to 320 $\mu \mathrm{M}$ when $5 \mathrm{mM} \mathrm{NaCl}$ with $145 \mathrm{mM}$ tetramethylammonium chloride (TMACl) is present in the buffer $(10 \mathrm{mM}$ Tris/ $\mathrm{HCl}$ with $0.1 \%$ PEG 6000 at $\mathrm{pH} 8$ in both cases) [23].

To detect a significant amount of protein complex, a relatively high excess of BPTI is necessary. Since $R_{\text {th }}$ and $R_{\mathrm{XL}}$ are relative measures related to the total amount of $\alpha$-thrombin in solution, the absolute concentration of $\alpha$-thrombin has little influence on both $\mathrm{R}$ values. In contrast, variations of the BPTI concentration cause a significant variation of R. For $\mathrm{K}_{\mathrm{D}}=320 \mu \mathrm{M}, \mathrm{R}_{\mathrm{th}}$ is about $5 \%$ for $20 \mu \mathrm{M}$ BPTI concentration, whereas at $100 \mu \mathrm{M}$ concentration a relative amount of $\alpha$-thrombin $\bullet$ BPTI of
$23 \%$ is expected. An $\alpha$-thrombin concentration of about $23 \mu \mathrm{M}$ was chosen to reduce the risk of signal suppression for $\alpha$-thrombin related signals. Surprisingly, few unspecific gas-phase adducts were observed in the MALDI mass spectra for the conditions chosen, which simplified spectra interpretation significantly. Crosslinking experiments were first performed at different BPTI concentrations ranging from 20 to $100 \mu \mathrm{M}$ in 10 $\mathrm{mM} \mathrm{Na} \mathrm{HPO}_{4} / \mathrm{NaH}_{2} \mathrm{PO}_{4}$ at $\mathrm{pH} 8.0$ with no additional salt. Significant amounts of cross-linked $\alpha$-thrombin $\bullet$ BPTI were detected above $40 \mu \mathrm{M}$. Cross-linking times of $4 \mathrm{~h}$ at room temperature yielded $\mathrm{R}_{\mathrm{XL}}$ values of about $50 \%$ of $\mathrm{R}_{\mathrm{th}}$ (data not shown). For longer reaction times of $24 \mathrm{~h}, \mathrm{R}_{\mathrm{XL}}$ even exceeded $\mathrm{R}_{\mathrm{th}}$ by $100 \%$, indicating unspecific complex formation for BPTI concentrations of 40 and $60 \mu \mathrm{M}$ (for higher concentrations spectra quality decreased dramatically after $24 \mathrm{~h}$ cross-linking due to the higher concentration of cross-linker present in solution). With the addition of $150 \mathrm{mM} \mathrm{NaCl}$ or 145 $\mathrm{mM}$ TMACl with $5 \mathrm{mM} \mathrm{NaCl}$ to the buffer, no significant changes of $R_{X L}$ were observed within error. All the described results indicate that at concentrations above $40 \mu \mathrm{M}$, nonspecific cross-linking is favored. Additionally, control experiments with the same BPTI concentrations but no $\alpha$-thrombin present in solution yielded cross-linked BPTI dimers, trimers, and tetramers after $24 \mathrm{~h}$ of cross-linking, whose extent could be correlated with the BPTI concentration. In summary, chemical cross-linking in combination with MALDI-MS is unsuitable for weak complexes with the experimental procedure described above.

\section{Conclusions}

The observed correlation between the binding affinity and the fraction of cross-linked complex in the midaffinity range suggests that chemical cross-linking in combination with MALDI-MS can be used to rank binding affinities. Specific complexes could be detected up to protein and peptide concentrations of $35 \mu \mathrm{M}$. However, for low-affinity complexes in the $10^{-4} \mu \mathrm{M} \mathrm{K}$ range, the simple protocol that includes the cross-linking step followed by direct analysis with MALDI-MS cannot be applied. A more sophisticated protocol would be necessary, using, for example, much lower concentrations for cross-linking and subsequent enrichment of the protein and protein-peptide complex. For all experiments performed in this work, comparable conditions for chemical cross-linking were utilized; i.e., comparable numbers of cross-linkable amino acids were present. Additionally, only one cross-linker, targeting mainly primary amines, was tested. However, the need for specific amino acids as targets for a certain cross-linking agent can restrict the application range of this method, especially when the chemical structure of the ligands is considerably different. To use chemical cross-linking and mass spectrometry to study the binding affinity of an unknown sample, experiments using a variety of cross-linkers with different spacer lengths and reactive 
groups have to be performed. For the measurements described herein, a 30- and 50-fold excess of cross-linker was used. When dealing with unknown protein complexes, optimization experiments to generate a maximum amount of cross-linked complex with a minimal crosslinker concentration are suggested.

We suggest that chemical cross-linking in combination with MALDI-MS could also rank binding affinities of proteins binding multiple ligands. The main prerequisite would again be a comparable availability of cross-linkable amino acids close to the binding interface. However, since this study is the first one dealing with chemical cross-linking and MALDI-MS over a broad range of binding affinities, the investigation of "higher-order complexes" is far beyond the scope of this work and will be the subject of future research.

\section{Acknowledgments}

The authors acknowledge financial support for this work by the Swiss National Science Foundation, grant no. 200020-124663. Additionally, the authors are thankful to Benoit Plêt and Alexis Nazabal, CovalX AG, Zurich, Switzerland for providing access and assistance to the Reflex III MALDI-TOF instrument, the group of Professor D. Hilvert, ETH Zurich, Switzerland for the use of the NanoDrop, and Matthias C. Jecklin for helpful discussions.

\section{References}

1. Kleanthous, C. Protein-Protein Recognition; Oxford University Press: Oxford, New York, 2000; p. 4

2. Vaynberg, J.; Qin, J. Weak Protein-Protein Interactions as Probed by NMR Spectroscopy. Trends Biotechnol. 2006, 24, 22-27.

3. Sinz, A. Chemical Cross-Linking and Mass Spectrometry for Mapping Three-Dimensional Structures of Proteins and Protein Complexes. J. Mass Spectrom. 2003, 38, 1225-1237.

4. Sinz, A. Chemical Cross-Linking and Mass Spectrometry to Map Three-Dimensional Protein Structures and Protein-Protein Interactions. Mass Spectrom. Rev. 2006, 25, 663-682.

5. Farmer, T. B.; Caprioli, R. M. Assessing the Multimeric States of Proteins: Studies Using Laser Desorption Mass Spectrometry. Biol. Mass Spectrom. 1991, 20, 796-800.

6. Farmer, T. B.; Caprioli, R. M. Determination of Protein-Protein Interactions by Matrix-Assisted Laser Desorption/Ionization Mass Spectrometry. J. Mass Spectrom. 1998, 33, 697-704.

7. Nazabal, A.; Wenzel, R.; Zenobi, R. Immunoassays with Direct Mass Spectrometric Detection. Anal. Chem. 2006, 78, 3562-3570.

8. Pimenova, T.; Pereira, C. P.; Schaer, D. J.; Zenobi, R. Characterization of High Molecular Weight Multimeric States of Human Haptoglobin and Hemoglobin-Based Oxygen Carriers by High-Mass MALDI MS. J. Sep. Sci. 2009, 32, 1224-1230.

9. Lomant, A. J.; Fairbanks, G. Chemical Probes of Extended Biological Structures: Synthesis and Properties of the Cleavable Protein CrossLinking Reagent [35S]Dithiobis(Succinimidyl Propionate). J. Mol. Biol. $1976,104,243-261$.
10. Madler, S.; Bich, C.; Touboul, D.; Zenobi, R. Chemical Cross-Linking with NHS Esters: A Systematic Study on Amino Acid Reactivities. J. Mass Spectrom. 2009, 44, 694-706.

11. Kalkhof, S.; Sinz, A. Chances and Pitfalls of Chemical Cross-Linking with Amine-Reactive N-Hydroxysuccinimide Esters. Anal. Bioanal. Chem. 2008, 392, 305-312.

12. Bich, C.; Scott, M.; Panagiotidis, A.; Wenzel, R. J.; Nazabal, A.; Zenobi, R. Characterization of Antibody-Antigen Interactions: Comparison Between Surface Plasmon Resonance Measurements and High-Mass Matrix-Assisted Laser Desorption/Ionization Mass Spectrometry. Analyt. Biochem. 2008, 375, 35-45.

13. Herzig, M. C. S.; Leeblundberg, L. M. F. The Agonist Binding-Site on the Bovine Bradykinin B2 Receptor Is Adjacent to a Sulfhydryl and Is Differentiated from the Antagonist Binding-Site by Chemical CrossLinking. J. Biol. Chem. 1995, 270, 20591-20598.

14. Gardsvoll, H.; Dano, K.; Ploug, M. Mapping Part of the Functional Epitope for Ligand Binding on the Receptor for Urokinase-Type Plasminogen Activator by Site-Directed Mutagenesis. J. Biol. Chem. 1999, 274, 37995-38003

15. Gardsvoll, H.; Gilquin, B.; Le Du, M. H.; Menez, A.; Jorgensen, T. J. D.; Ploug, M. Characterization of the Functional Epitope on the Urokinase Receptor - Complete Alanine Scanning Mutagenesis Supplemented by Chemical Cross-Linking. J. Biol. Chem. 2006, 281, 19260-19272.

16. Bovet, C.; Ruff, M.; Eiler, S.; Granger, F.; Wenzel, R.; Nazabal, A.; Moras, D.; Zenobi, R. Monitoring Ligand Modulation of Protein-Protein Interactions by Mass Spectrometry: Estrogen Receptor $\alpha$-SRC1. Anal. Chem. 2008, 80, 7833-7839.

17. Vidal-Madjar, C.; Jaulmes, A.; Renard, J.; Peter, D.; Lafaye, P. Chromatographic Study of the Adsorption Kinetics of Albumin on Monoclonal and Polyclonal Immunoadsorbents. Chromatographia 1997, 45, 18-24.

18. Schulz, D. M.; Ihling, C.; Clore, G. M.; Sinz, A. Mapping the Topology and Determination of a Low-Resolution Three-Dimensional Structure of the Calmodulin-Melittin Complex by Chemical Cross-Linking and High-Resolution FTICRMS: Direct Demonstration of Multiple Binding Modes. Biochemistry 2004, 43, 4703-4715.

19. Frickel, E. M.; Riek, R.; Jelesarov, I.; Helenius, A.; Wuthrich, K. Ellgaard, L. TROSY-NMR Reveals Interaction Between ERp57 and the Tip of the Calreticulin P-Domain. Proc. Natl. Acad. Sci. U.S.A. 2002, 99, 1954-1959.

20. Litterst, C. M.; Pfitzner, E. An LXXLL Motif in the Transactivation Domain of STAT6 Mediates Recruitment of NCoA-1/SRC-1. J. Biol. Chem. 2002, 277, 36052-36060.

21. Green, N. S.; Reisler, E.; Houk, K. N. Quantitative Evaluation of the Lengths of Homobifunctional Protein Cross-Linking Reagents Used as Molecular Rulers. Protein Sci. 2001, 10, 1293-1304.

22. Seitz, M.; Maillard, L. T.; Obrecht, D.; Robinson, J. A. Molecular Characterization of the NCoA-1-STAT6 Interaction. Chem. Biochem. 2008, 9, 1318-1322.

23. De Cristofaro, R.; Landolfi, R. Allosteric Modulation of BPTI Interaction with Human $\alpha$ - and $\zeta$-Thrombin. Eur. J. Biochem. 1999, 260, 97-102.

24. Wenzel, R.; Roehling, U.; Nazabal, A.; Hillenkamp, F. A. Detector Device for High Mass Ion Detection, a Method for Analyzing Ions of High Mass and a Device for Selection Between Ion Detectors. 2009.

25. Gruic-Sovulj, I.; Ludemann, H. C.; Hillenkamp, F.; Weygand-Durasevic, I.; Kucan, Z.; Peter-Katalinic, J. Detection of Noncovalent tRNAAminoacyl-tRNA Synthetase Complexes by Matrix-Assisted Laser Desorption/Ionization Mass Spectrometry. J. Biol. Chem. 1997, 272, $32084-$ 32091.

26. Behrendt, N.; Ronne, E.; Dano, K. Domain Interplay in the Urokinase Receptor-Requirement for the Third Domain in High Affinity Ligand Binding and Demonstration of Ligand Contact Sites in Distinct Receptor Domains. J. Biol. Chem. 1996, 271, 22885-22894.

27. Bich, C.; Baer, S.; Jecklin, M. C.; Zenobi, R. Probing the Hydrophobic Effect of Noncovalent Complexes by Mass Spectrometry. J. Am. Soc. Mass Spectrom. 2010, 21, 286-289.

28. Bich, C.; Maedler, S.; Chiesa, K.; DeGiacomo, F.; Bogliotti, N.; Zenobi, R. Reactivity and Applications of New Amine Reactive Cross-Linkers for Mass Spectrometric Detection of Protein-Protein Complexes. Anal. Chem. 2010, 82, 172-179.

29. Wells, C. M.; Dicera, E. Thrombin Is a Na+-Activated Enzyme. Biochemistry 1992, 31, 11721-11730. 